\title{
Proliferative myositis in the right brachioradialis: A case report
}

\author{
NA WEI ${ }^{1}$, WEI-JIE XU ${ }^{2}$, DONG DONG ${ }^{3}$ and YU-BAO GONG ${ }^{2}$ \\ ${ }^{1}$ The First Operating Room; Departments of ${ }^{2}$ Bone and Joint Surgery and ${ }^{3}$ Radiology, \\ The First Hospital of Jilin University, Changchun, Jilin 130000, P.R. China
}

Received May 7, 2015; Accepted February 27, 2017

DOI: $10.3892 /$ etm.2017.4269

\begin{abstract}
Proliferative myositis is a rare, self-limiting, benign disease. Its diagnosis can be difficult and in many cases is not confirmed until after surgical resection. Herein, we report a case of proliferative myositis of the right brachioradialis in a 64-year-old man. The patient presented with a rapidly growing, painless mass in his right forearm. Magnetic resonance imaging and fine-needle aspiration biopsy led to a diagnosis of proliferative myositis. Complete surgical resection of the mass was performed. Postoperative pathological examination confirmed the diagnosis. To the best of our knowledge, this is the first report of proliferative myositis in the right brachioradialis. Fine-needle biopsy is helpful in the diagnosis of proliferative myositis, thus avoiding unnecessary surgical trauma and costs.
\end{abstract}

\section{Introduction}

Proliferative myositis is a rare pseudosarcomatous (mimicking sarcoma) condition. It usually occurs in adults aged $>40$-years-old, and occasionally in children (1). Although proliferative myositis grows rapidly, it is actually a benign lesion and can regress or completely resolve spontaneously. The incidence of proliferative myositis remains unknown, however recurrence is extremely rare (2). Although the cause of proliferative myositis remains unclear, a history of recent local trauma may be a risk factor, as noted in some reports $(1,3,4)$. Diagnosis of proliferative myositis is largely dependent on the patient's age, history of recent trauma, and a rapidly growing, painful solitary soft-tissue mass located in the muscle, together with radiological evidence, such as magnetic resonance imaging (MRI). Pathological examination of a biopsy is the final and most accurate method for the diagnosis of proliferative myositis. There is no specific treatment recommended following establishment of a diagnosis of proliferative myositis, since of proliferative myositis may disappear spontaneously $(2,5)$. Excision may be

Correspondence to: Dr Yu-Bao Gong, Department of Bone and Joint Surgery, The First Hospital of Jilin University, 71 Xinmin Street, Changchun, Jilin 130000, P.R. China

E-mail: gongyubao@hotmail.com

Key words: brachioradialis, magnetic resonance imaging, proliferative myositis, pathology, resection preferred for the confirmation of a diagnosis and for cosmetic reasons (2). The most commonly involved sites are the head, neck, and extremities. Herein, we report a case of proliferative myositis in the right brachioradialis. To the best of our knowledge, this is the first case reported at this site and will further improve our understanding of the sites affected by this disease.

\section{Case report}

A 64-year-old man was admitted to The First Hospital of Jilin University (Changchun, China) in September 2014 after presenting with a mass in his right forearm. The mass was incidentally found by the patient 2 weeks previously, and upon hospitalization had grown larger. On examination, a mass $3.0 \times 2.5 \mathrm{~cm}$ was palpated in the right brachioradialis, on the lateral side of the proximal end of the right forearm. During movement and rest of his right arm, the patient experienced no discomfort. The skin surface above the mass was elevated, and exhibited normal pigmentation and temperature. The mass was mildly tender with a hard texture and smooth surface, although the border with the surrounding tissue was not pronounced.

Neurological examination of the right arm was unremarkable. MRI showed a hyperintense lesion in T1- and T2-weighted images (Fig. 1). Fine-needle biopsy of the lesion revealed spindle cells and ganglion cells, suggesting proliferative myositis. Pathological examination was subsequently performed. The specimen was fixed in $10 \%$ formalin-saline solution, followed by embedding in paraffin for $24 \mathrm{~h}$, sectioning at $4-\mu \mathrm{m}$ thickness, and staining with hematoxylin and eosin. Subsequently, the sections were examined under a light microscope (DP20; Olympus Corp., Tokyo, Japan) at a magnification of $x 40$. Since pathological examination demonstrated that the lesion was benign and the function of the arm was not affected, a watch-and-wait strategy was recommended. However, the patient insisted on surgical resection of the mass.

Intraoperatively, the mass was discovered to be incorporated with surrounding muscular tissues. Complete resection of the mass was performed with dissection of the surrounding normal tissues.

Gross pathological examination showed that the resected mass was pink-gray on the transverse section surface (Fig. 2A). Microscopic examination revealed ganglion-like giant basophilic cells and spindle cells (Fig. 2B and C). A diagnosis of proliferative myositis was confirmed pathologically. Upon follow-up 13 months after surgery, the patient exhibited a well-healed surgical incision and good elbow joint function, 

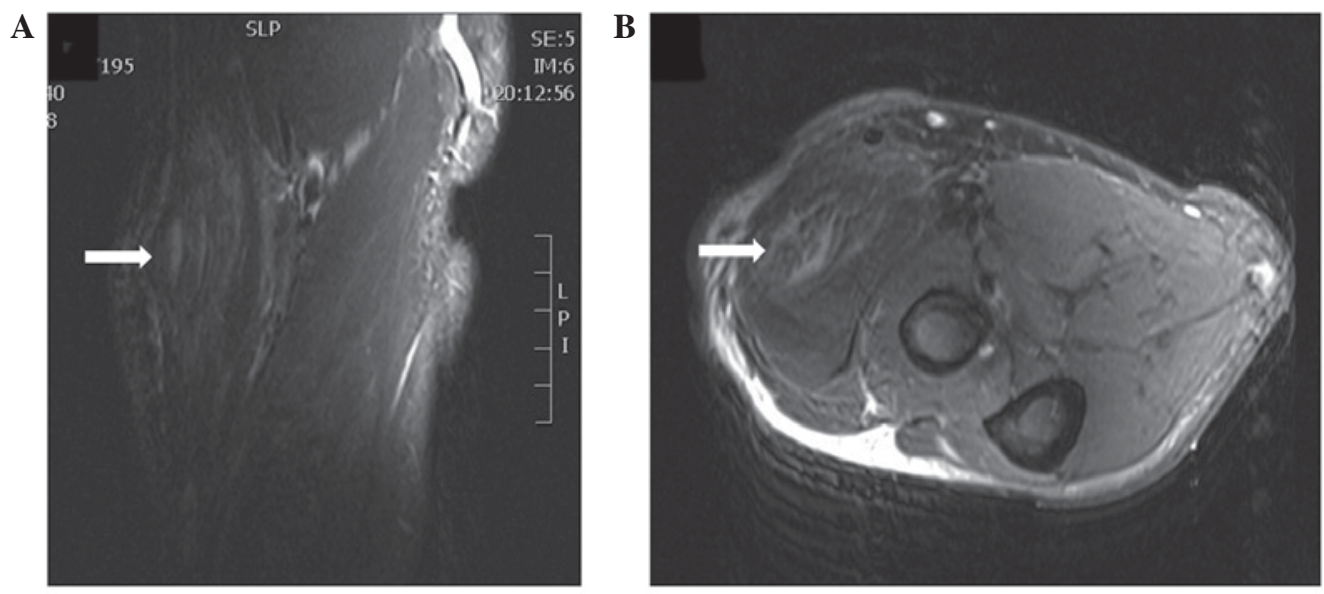

Figure 1. T2-weighted magnetic resonance images with fat suppression. (A) Coronal and (B) axial views. Arrows denote the lesion in the right brachioradialis.

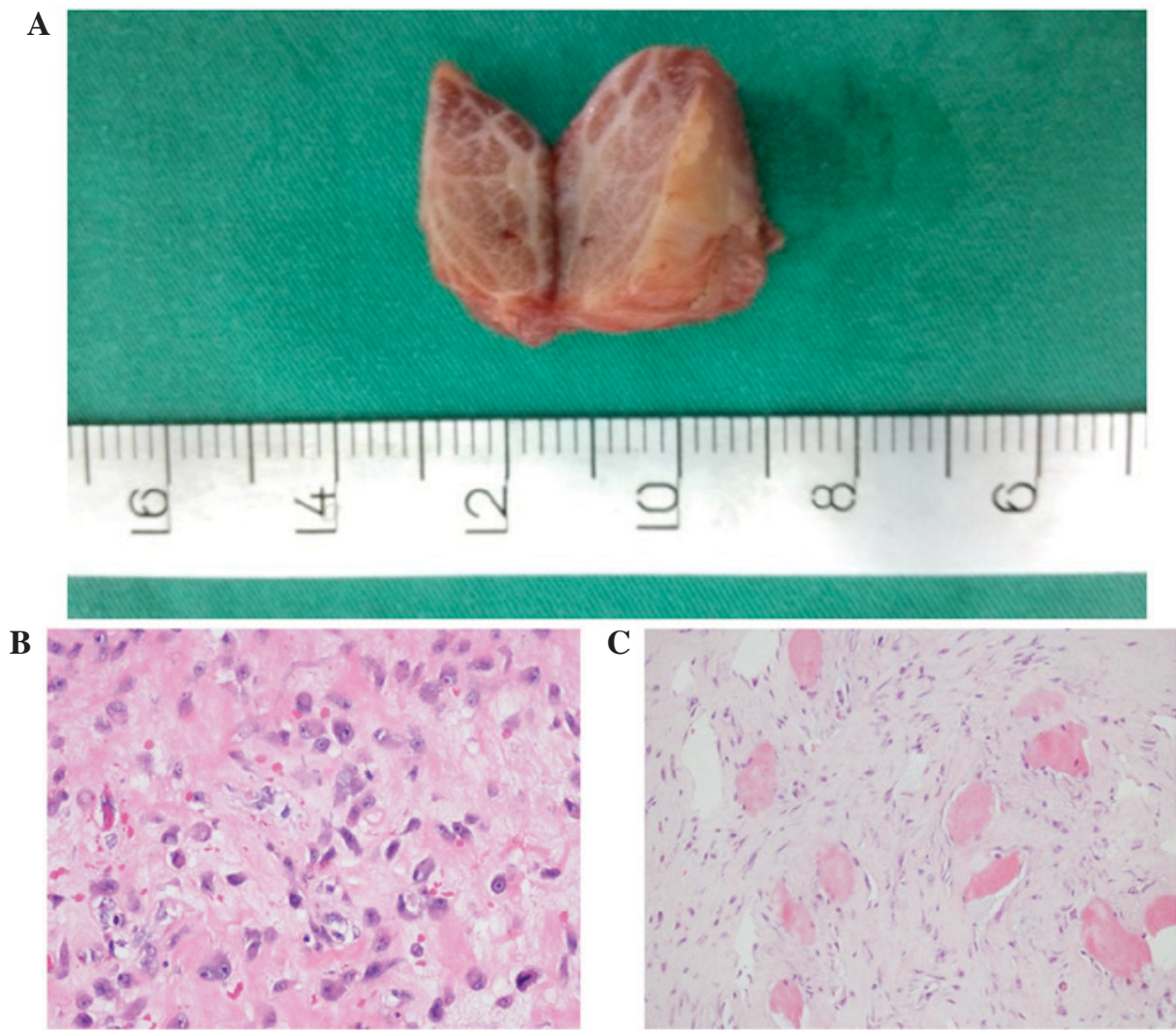

Figure 2. Pathological examination of the resected proliferative myositis. (A) The mass was pink-gray on the transverse section surface with a solid texture, integrated with the surrounding muscle. (B) Ganglion-like giant basophilic cells were detected and (C) muscle bundles were divided by the spindle cells, in a checkerboard-like pattern, as detected by hematoxylin and eosin staining (magnification, $\mathrm{x} 40$ ).

without any swelling or tenderness. No recurrence of the mass was detected. The patient refused a follow-up MRI examination.

Ethical approval for the present study was obtained from The First Hospital of Jilin University and written informed consent was obtained from the patient.

\section{Discussion}

Proliferative myositis was first reported by Kern in 1960 (2). Several theories regarding its etiology have been proposed, such as mechanical injury and ischemia, which remain inconclusive (6-9). The mass usually grows rapidly and is able to cause local compression symptoms.

Proliferative myositis shows no specific features upon laboratory and imaging examinations; therefore, pathological examination is required for a definitive diagnosis. The resected mass is lobular and infiltrative. Its section surface is gray in color, with a solid texture. Pathologically, the proliferative mass is characterized by a checkerboard of myofibroblasts infiltrating muscle fibers on the transverse section, and ganglion-like cells (10). 
Proliferative myositis is distinct from rhabdomyosarcoma, myositis ossificans, nodular fasciitis, and proliferative fasciitis $(2,11,12)$. Differentiating between proliferative myositis and rhabdomyosarcoma can be difficult. Soft tissue sarcoma is often round in shape or nodular, lacking the normal structure of muscle fibers. In large soft tissue sarcomas, necrosis, hemorrhage, and cysts may be found (12). In addition, proliferative myositis is negative for myosin staining, but positive in rhabdomyosarcoma. Myositis ossificans exhibit characteristic ossification in the lesion, as observed on MRI or computed tomography (10). MR images of proliferative myositis have been reported in several cases, with hypo- or iso-intense T1 signals, compared with those of muscle and homogeneous enhancement. T2-weighted MR images typically demonstrate a hyperintense soft-tissue mass $(1,2,5)$.

A watch-and-wait strategy is preferred for proliferative myositis, due to its benign nature and the potential for spontaneous resolution. Although the mass grows rapidly in its initial phase, it typically stabilizes after a few weeks. No malignancy or metastasis of proliferative myositis has ever been reported (9). If the mass is causing compression symptoms or affecting the patient's daily life, surgical resection can be performed.

In conclusion, proliferative myositis is a rare, self-limiting, benign disease that has not been described in the right brachioradialis until now. Its diagnosis can be difficult and, in many cases, diagnosis is not confirmed until after surgical resection. Fine-needle biopsy is helpful in the diagnosis of proliferative myositis, thus avoiding unnecessary surgical trauma and costs.

\section{References}

1. Mulier S, Stas M, Delabie J, Lateur L, Gysen M, Dal Cin P, Robberecht $\mathrm{C}$ and De Wever I: Proliferative myositis in a child. Skeletal Radiol 28: 703-709, 1999.

2. Wlachovska B, Abraham B, Deux JF, Sibony M, Marsault C and Le Breton C: Proliferative myositis in a patient with AIDS. Skeletal Radiol 33: 237-240, 2004.

3. Kern WH: Proliferative myositis; a pseudosarcomatous reaction to injury: A report of seven cases. Arch Pathol 69: 209-216, 1960.

4. Enzinger FM and Dulcey F: Proliferative myositis: Report of thirty-three cases. Cancer 20: 2213-2223, 1967.

5. Kent MS, Flieder DB, Port JL, Altorki NK. Proliferative myositis: a rare pseudosarcoma of the chest wall. Ann Thorac Surg 2002;73:1296-1298.

6. Wong NL: Fine needle aspiration cytology of pseudosarcomatous reactive proliferative lesions of soft tissue. Acta Cytol 46: 1049-1055, 2002.

7. Wong NL and Di F: Pseudosarcomatous fasciitis and myositis diagnosisby fine-needle aspiration cytology. Am J Clin Pathol 132: 857-865, 2009.

8. Brooks JK, Scheper MA, Kramer RE, Papadimitriou JC, Sauk JJ and Nikitakis NG: Intraoral proliferative myositis: Case report and literature review. Head Neck 29: 416-420, 2007.

9. Fauser C, Hrig J, Niedermeyer HP and Arnold W: Proliferative myositisa rare pseudomalignant tumor of the head and neck. Arch Otolaryngol Head Neck Surg 134: 437-440, 2008.

10. Klapsinou E, Despoina P and Dimitra D: Cytologic findings and potential pitfalls in proliferative myositis and myositis ossificans diagnosed by fine needle aspiration cytology: Report of four cases and review of the literature. Diagn Cytopathol 40: 239-244, 2012.

11. Subramanian S and Sharma R: Can MR imaging be used to reliably differentiate proliferative myositis from myositis ossificans? Radiology 246: 987, 2008.

12. Demir MK, Beser M and Akinci O: Case 118: Proliferative myositis. Radiology 244: 613-616, 2007. 